\title{
El boxeador Zhang Songxi y los orígenes del concepto de Escuela Interna-Externa
}

Marnix W ells ${ }^{1} \&$ Stanley E. Henning ${ }^{2}$

${ }^{1}$ Tai Chi Union (Reino Unido). ${ }^{2}$ nnvestigador independiente (Estados Unidos).

Es traducción del artículo Boxer Zhang Songxi and the Origins of the Internal-External School Concept, publicado en el Journal of Asian Martial Arts, volumen 20, número 2

(22-29), 2011

\section{Resumen}

Zhang Songxi era tan respetado por sus habilidades pugilísticas que inspiró al secretario mayor del emperador, Shen Yiguan (1531-1616), a escribir la Biografía del Boxeador Zhang Songxi. Más tarde, el historiador Huang Zongxi (1610-1695) escribió un epitafio para un luchador de la resistencia llamado W ang Zhengnan. En la biografía etiquetó tanto a W ang como a Zhang como practicantes de una "escuela interna de boxeo", en comparación con lo que él Illamaba Shaolin o "escuela externa". Puesto que Shen Yiguan nunca mencionó a Zhang como practicante de la escuela interna, parece que Huang estaba utilizando el concepto de interno-externo para expresar simbólicamente su insatisfacción con los gobernantes manchúes de China. La biografía de Zhang Songxi proporciona algunas evidencias de que el concepto de escuela interna-externa estaba orientado más políticamente que hacia las artes marciales.

Palabras clave: China, artes marciales chinas, estilos internos, estilos externos.

Boxer Z hang Songxi and the Origins of the Internal-External School Concept Abstract: Zhang Songxi was so highly respected for his boxing skills that the emperor's grand secretary, Shen Yiguan (1531-1616), was inspired to write the Biography of Boxer Zhang Songxi. Later the historian Huang Zongxi (1610-1695) wrote an epitaph for a resistance fighter named W ang Zhengnan. In the biography he labeled both W ang and Zhang as practitioners of the "internal school of boxing," versus what he called the Shaolin or "external school." Since Shen Yiguan never mentioned Zhang as an internalschool practitioner, it appears that Huang was using the internal-external concept to symbolically express his dissatisfaction with China's Manchu rulers. The Zhang Songxi biography provides some evidence that the internal-external school concept was more political than martial arts oriented.

Key words: China, Chinese martial arts, internal styles, external styles. 


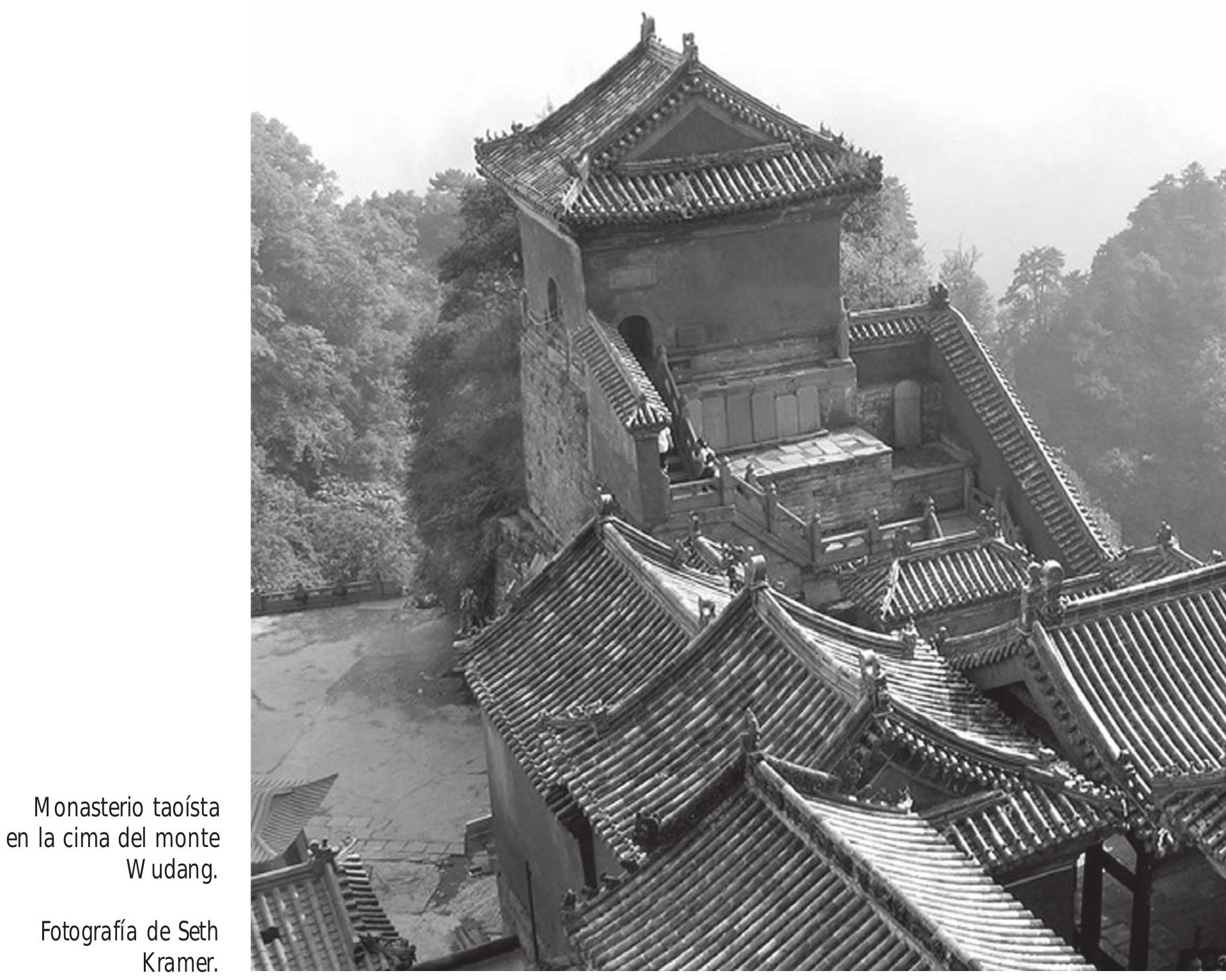

0 boxeur Zhang Songxi e as origens do conceito da Escola Interna-Externa

Resumo: Zhang Songxi é tão respeitado pelas suas habilidades pugilistas que inspirou 0 secretário-geral do Imperador, Shen Yiguan (1531-1616), a escrever a Biografia do Boxeur Zhang Songxi. Mais tarde, o historiador Huang Zongxi (1610-1695) escreveu um epitáfio para um lutador da resistência chamado W ang Zhengnan. Na biografia, classificou W ang e Zhang como praticantes de uma "escola interna de boxe", em comparação com o que ele chamava de Shaolin ou "escola externa". Dado que Shen Yiguan nunca mencionou Zhang como praticante da escola interna, parece que Huang estava utilizando o conceito de interno-externo para expressar simbolicamente a sua insatisfação com os governantes manchus da China. A biografia de Zhang Songxi proporciona algumas evidências de que o conceito da escola interna-externa estava mais orientado politicamente que para as artes marciais.

Palavras-chave: China, artes marciais chinesas, estilos internos, estilos externos. 
da del término "nei jia" en relación a las artes marciales chinas. Desde entonces, mucho se ha escrito sobre las escuelas internas, pero recientes investigaciones nos están forzando a cuestionar el significado y fundamentos de hacer tal distinción entre internas y externas. La primera biografía conocida de Zhang Songxi ha salido recientemente a la luz; ese trabajo proporciona la mayor parte de los detalles que presentamos en este artículo.

Shen Yiguan (1531-1615) escribió la biografía durante los últimos años de la dinastía Ming (1368-1644). Escrita aproximadamente 50 años antes que la de Huang Zongxi, Shen no muestra ningún conocimiento sobre la terminología nei jia utilizada en las tradiciones de combate, ni tampoco menciona ninguna otra posible conexión taoísta. Sin embargo, compara el estilo de boxeo refinado de Zhang con la fuerza bruta empleada por los monjes Shaolin.

Como secretario mayor, Shen ostentaba una alta posición política en un momento donde las condiciones se estaban deteriorando rápidamente en China. Escribió la Biografía del boxeador Zhang Songxi (Zhang, 1988; Zhang, 1932) mientras China estaba siendo humillada por la opresión extranjera. Los japoneses habían invadido Corea con planes para avanzar hacia China, pero fueron los ejércitos manchúes los que conquistaron Beijing en 1644. Shen estaba motivado para escribir la biografía por el interés en un conciudadano y en las cuestiones militares/marciales de las que muestra alguna comprensión desde una obvia perspectiva confuciana. Su posición única - ligada a la política y las cuestiones militares- tuvo influencia en su redacción de la biografía de Zhang Songxi.

El escrito de Shen se centra tanto en el carácter moral de Zhang como en sus habilidades marciales. Para ilustrar este equilibrio, podemos echar un vistazo a algunas de las historias recogidas por Shen Yiguan. Además observaremos en detalle lo que se conoce como el "código de cinco palabras" de Zhang, una fórmula esotérica para obtener unas habilidades pugilísticas de nivel superior. Existe una similitud asombrosa entre los informes de Shen y Huang sobre el código de cinco palabras de Zhang, aunque difieren en sus interpretaciones.

\section{Los monjes Shaolin se encuentran con Zhang Songxi}

Shen Yiguan compara a Zhang Songxi con un monje marcial Shaolin de la época llamado Bian Cheng, que es descrito como duro y brutal, mientras que Zhang era calmado, decidido y cortés como un erudito. Shen relata que durante el periodo de los ataques piratas japoneses a mediados del s. XVI, un grupo de setenta monjes Shaolin o más buscó a Zhang para un combate de habilidades. Trató de evitarlos hasta que un grupo de jóvenes le convencieron para ir con ellos a la posada donde los monjes se alojaban para observar su práctica.

Cuando Zhang y los jóvenes rompieron a reír ante lo que estaban contemplando, los monjes los interceptaron y Zhang fue acorralado. Zhang era pequeño de estatura y los monjes eran grandes y fuertes. Zhang se sentó en una silla, con las manos en sus mangas, frente a una ventana del segundo piso de la posada donde se alojaban los monjes. Uno de ellos saltó hacia Zhang, que se giró en la silla, levantó su mano y envió al monje volando por la ventana hacia el suelo, no matándole por poco. Zhang puso en duda la habilidad del monje, apuntando que elevar los pies en boxeo era un enfoque ineficaz fácil de contraatacar. 


\section{El enfoque de $Z$ hang del empuje en el combate}

En cierto momento, un oficial de reclutamiento intentó alistar a Zhang para que entrenase a los soldados, pero él se negó, alegando que había prometido a su maestro que no enseñaría a hombres de carácter cuestionable. Sólo dio clases a una pareja de estudiantes. Uno de ellos con quién Shen se encontró dijo esto del enfoque de Zhang: " $i$ Empujar es empujar! Si gastas demasiado tiempo en la reflexión mental, perderás el objetivo. ¿Cómo puedes estar entonces centrado?"

Shen entonces recordó que hace mucho tiempo había preguntado al general Wang Zhongbo sobre las tropas fronterizas: "¿Qué habilidades tienen que les hacen tan buenos para la guerra?". Zhongbo respondió: "Las tropas fronterizas no tienen habilidades. Esperan a que los bárbaros se acerquen a treinta pasos antes de lanzar sus flechas. Cuando las armas cortas chocan, van directamente hacia delante para atacar y empujar. No miran a izquierda 0 derecha, y así vencen. ¡El que parpadea no sabe; el que mira a un lado está muerto!". Shen se dio cuenta de que Zhang aplicaba este mismo principio.

Más tarde Shen comparaba esto con el cultivo del coraje del antiguo héroe You Beigong, como lo describe el filósofo Mencio. Estaba escrito que, frente al combate, la carne de You no se estremecía y sus ojos no parpadeaban. Esto no significa que los hombres no le empujasen. En cuanto a lo de estremecerse y parpadear, era simplemente como moscas posándose en su cuerpo. Olvidó estremecerse y parpadear. Despertó su esencia y empleó sus energías, concentrándose con firmeza sin ninguna ambigüedad (Legge, 1961). Shen recuerda que en el periodo Tang (618-907) la cara del general Lei Wanchun estaba perforada por siete flechas, y que él no se movía. Lei murió heroicamente por el

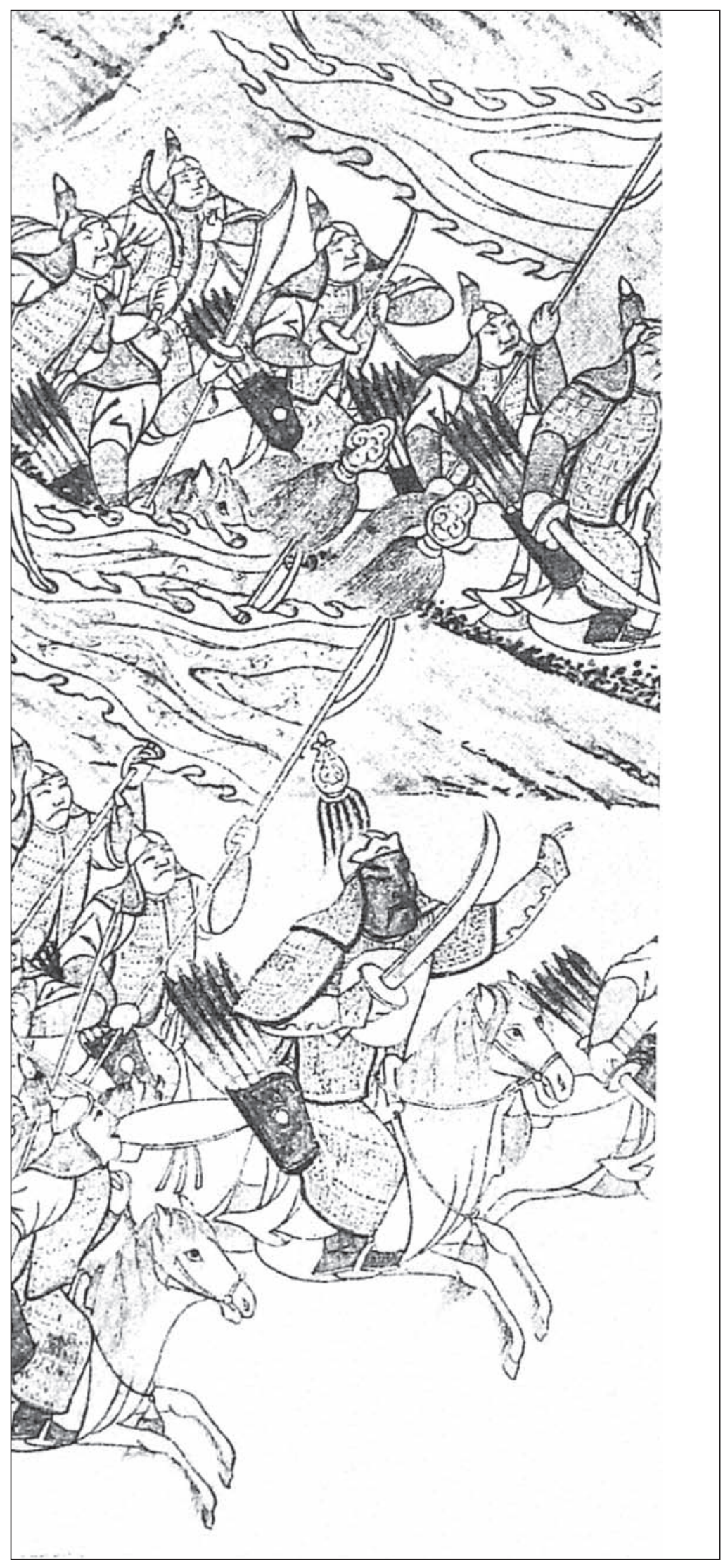

Conquista manchú de China. 
emperador Tang en la muralla fortificada de la ciudad de Suiyang en la provincia de Henan, resistiendo contra la devastadora rebelión de An Lushan en los años 755-756. Shen era consciente de que este "coraje" mantenía el espíritu del "empuje" de Zhang.

\section{Zhang no era un actor}

Para Shen, Zhang personificaba el verdadero guerrero que es reacio a mostrar su fuerza. Una vez Zhang fue al campo a dar un paseo, fuera de la ciudad. Varios jóvenes le invitaron a mostrar sus habilidades pugilísticas, pero Zhang se negó rotundamente. Cuando regresó a la puerta de la ciudad, los jóvenes dijeron al guardián que no dejase entrar a Zhang. Fue encerrado dentro de la puerta amurallada en forma de media luna (las ciudades principales tenían un espacio de entrada cercado, en forma de media luna, donde se podía atrapar a un invasor). Se inclinaron hacia Zhang, diciendo: "Ahora no puedes ir hacia delante ni hacia atrás. Como humildes espectadores te rogamos que hagas una exhibición para nosotros".

Zhang no tenía otra alternativa sino consentir. La puerta tenía varias piedras de rodillo que pesaban varios cientos de libras. Dijo a los jóvenes que las apilasen. Se las arreglaron para apilar hasta dos, pero no podían equilibrarlas. Zhang las sostuvo, colocó una pieza de baldosa sobre ellas, y luego añadió otra piedra más. Les suplicó, diciendo: "Soy una persona de setenta años sin ninguna utilidad. Si las parto en dos hasta abajo para haceros reír, ise arreglará todo?". Levantó su mano izquierda oblicuamente y golpeó. Cada una de las tres piedras se partió en dos.

Zhang raramente mostraba sus habilidades de lucha. Nunca se casó y no tuvo un hijo al que enseñar. Sólo dio clases a unos pocos individuos. Era un buen hijo y sirvió a su madre hasta su muerte.

\section{El código de cinco palabras de Zhang: una fórmula para obtener habilidades marciales}

Zhang elaboró un código de cinco palabras (五字訣 wu-zi jue) que condensaba sus enseñanzas en un formato sencillo de seguir para sus discípulos. Sus pocos discípulos valoraban la fórmula secreta, y casi nunca compartían las enseñanzas, excepto con aquellos en los que podían confiar ciegamente. El código consistía en estas cinco palabras: diligencia, intensidad o seguridad, franqueza, respeto y seriedad. Parafraseando a Shen, ofrecemos esta interpretación:

- Diligencia (勤 qin): Mañana y noche, trabaja para entrenar la fuerza de manos y pies. Reduce las horas de sueño. Recoge leña y agua, y prepara tú mismo las comidas. Amplía los propios esfuerzos a las llanuras centrales (la nación), sé siempre temeroso de escoger las cosas fáciles, y da el cien por cien.

2 Intensidad (紧 jin): Ambas manos deben proteger el centro del pecho y, en movimiento, uno debe protegerse las costillas, tanto por la izquierda como por la derecha. Cuando se golpea o se empuja, no agotes tu movimiento, 
de modo que puedas retroceder. Los pies deben apretarse con fuerza, como si estuviesen en una vía. No eleves alto tus pies 0 des un paso ancho. Deberían ser "como una $\mathrm{T}$, pero no una $\mathrm{T}$; como una $\mathrm{V}$, pero no una $\mathrm{V}$ ( $\mathrm{T}$ 不丁，八不八 ding bu ding, ba bu ba; los pies colocados para mantener el equilibrio). Podrás avanzar 0 retroceder rápidamente. Deja que tu mente esté constantemente inquieta y alerta, y no dejes que tu atención se apague. Tu postura debería ser estable. No dejes que tu pie de atrás esté sin peso. Todos los sentidos unidos en concierto; un centenar de articulaciones concentradas juntas. Enróllate como un erizo. Agazápate como un tigre. Esto es lo que las tácticas militares llaman "Ser como una virgen". Cuando el enemigo abre la puerta, es ventajoso acercarse a él.

3 Franqueza (經 jing): Esto es lo que se llama "Después conviértete en una liebre asustada". Demasiado lejos o no lo suficientemente lejos, y perderás. No hagas otros planes. No mires atrás. No pierdas el momento. Tienes que golpear el centro de la articulación. Una vez que has centrado tu deseo en un lugar, entonces agota cada poro de la fuerza de tu cuerpo para que todos avancen juntos sin la menor discrepancia, como un "gato cogiendo un ratón".

De este modo, en estas tres palabras - diligencia, intensidad y franqueza- las técnicas de golpeo y empuje se agotan. Zhang Songxi sólo recibió las tres primeras palabras de su maestro, que se llamaba Sun decimotercero. Las dos palabras finales en tono moralizante -respeto y seriedad- fueron añadidas por Zhang.

4 Respeto (敬 jing): Sé reverente y contente. No muestres tus fortalezas. Aquel que ama el desafío encontrará sin duda su combate. Ten cuidado. ¡Ten cuidado! Sé amable y bueno, modesto y flexible. No seas envidioso 0 ambicioso. Cada función está en reserva.

(5 Seriedad (切 qie): Resiste mil veces. ¡Resiste un sinfín! Cierra tus dedos. A prieta tus dientes. No permitas el desastre. No esperes la buena suerte. No dejes que tu cuerpo se enrede ligeramente con los hombres. La suerte y la mala suerte afectan a tu cuerpo. Solo cuando no queda otra alternativa deberías rebelarte. Si puedes retirarte después de un combate, entonces retírate. No deberías competir de nuevo. Incluso si en toda tu vida no puedes mostrar tu forma y nunca te haces famoso, no hay nada que lamentar. Si siembras enemistad, el mal karma nunca desaparecerá. Si rompes las normas y reglas del rey, tu sentencia será interminable. ¿No puede uno tener cuidado?

\section{La perspectiva confuciana de Shen Yiguan sobre las habilidades marciales}

La prudencia de Shen se muestra en sus escritos. Apuntaba que los verdaderos caballeros hacen sus cascos y armaduras de fe y lealtad, y sus torres de vigilancia de decencia y rectitud. Él pregunta: "¿No son estas suficientes defensas? 
En vez de meter miedo a los hombres y defenderse de ellos, ino es mejor no atemorizar a los hombres y así no necesitar defenderse? ¿No es esto más seguro?".

En otra parte Shen continúa: "Ahora el estudio de las habilidades es defenderse contra el desastre. Si tienes en cuenta la calamidad, tanto más crece. Entonces, ¿de qué sirven las habilidades?... Si dependes de tus habilidades, pero no anticipas el problema, jentonces el problema te encontrará! Así es difícil de justificar la confianza en las habilidades. De esta manera los caballeros respetan la virtud en lugar de enfatizar sus habilidades marciales"1.

En conjunto, la Biografía del boxeador Zhang Songxi de Shen refleja la combinación perfecta de defensa personal, auto-respeto y responsabilidad social que se esperaban dentro de las normas sociales confucianas respecto al estatus de una persona en la sociedad. Recomienda mantener el concepto confuciano expresado en el Registro de Historia: "He oído que aquellos versados en asuntos civiles tienen que estar militarmente preparados y aquellos versados en asuntos militares tienen que ser expertos en asuntos civiles" (Takigawa, 1972: 731).

Confirmando esta actitud está el hecho de que las estatuas e ilustraciones de Confucio a menudo le muestran a él y a sus seguidores portando espadas. La educación confuciana enfatizaba el estudio de las seis artes, que incluían el tiro con arco y la conducción de carros. Después del periodo de los Reinos Combatientes (476-221) la guerra masiva con carros se volvió obsoleta y se reemplazó

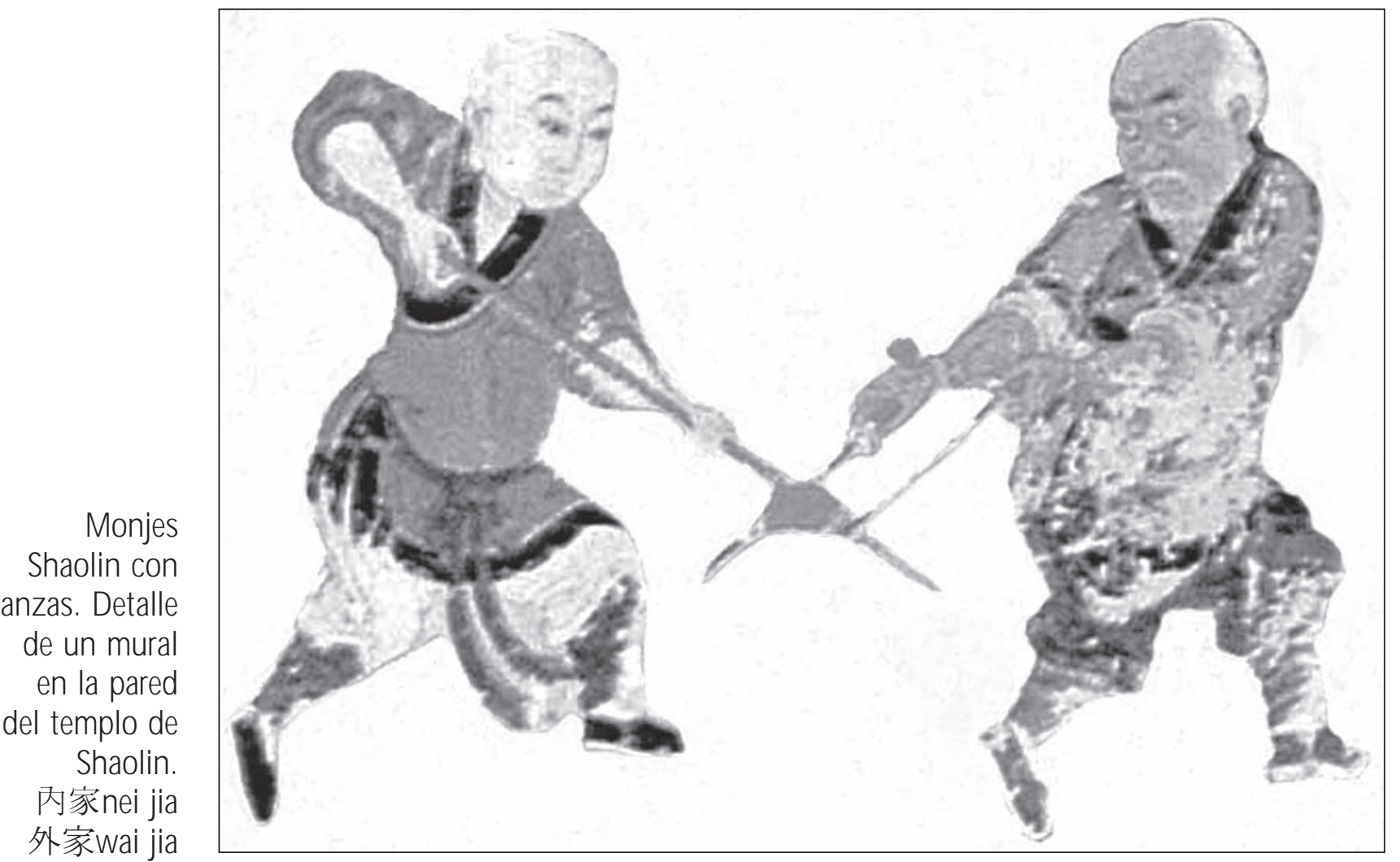

1 Literalmente, "deshacerse de las anteriores (habilidades) y mantener esta (virtud)". Mientras que Zhang Songxi trataba aparentemente su boxeo con una considerable reserva, los puntos de vista expresados aquí parecen ser más los del autor, Shen Yiguan. 
por la caballería y el tiro con arco a caballo. El tiro con arco a pie y a caballo continuó siendo testado en los exámenes marciales hasta que estos se suprimieron en 1901 (Zi, 1896).

Shen Yiguan no era uno de esos como Gu Yanwu (1613-1682) y otros que elogiaban la participación de los monjes Shaolin contra los piratas japoneses (Gu, 1928). Como un contemporáneo de las proximidades de la provincia de Fujian, Xie Zhaozhe (1567-1624), guardaba un punto de vista más crítico. Xie apuntó que, a pesar de que los monjes de Shaolin eran famosos por su boxeo, fueron asesinados en una emboscada, mostrando que las habilidades marciales no eran tan importantes como un liderazgo eficaz (Xie, 1935).

El código de cinco palabras de Zhang, como lo interpreta Shen, se refiere en dos ocasiones a las enseñanzas marciales que se encuentran en la historia de "La Doncella de Yue" ${ }^{\prime 2}$, que se conserva en un romance histórico de la última parte del periodo Han (25-220). Esto deja un cierto sabor místico en el arte, aunque Shen nunca utiliza el término "interno". De hecho, podría decirse que el enfoque de Zhang fue "internalizado" en el sentido de mantener sus técnicas secretas y no mostrarlas al público. Huang fue el primero en la historia en utilizar los términos "interno" y "externo" con un sentido sectario de lo taoísta frente a lo budista, y Wudang frente a Shaolin, con las implicaciones racistas de lo chino frente a lo foráneo. Huang era un ferviente nacionalista y un líder de la resistencia anti manchú de la provincia de Zhejiang. Se identificaba claramente con los luchadores nacionales de su provincia como Zhang Songxi y, en su propia época, W ang Zhengnan. Para él Shaolin, budismo y los conquistadores manchúes eran todos extranjeros, y por lo tanto "externos" (wai) -que también es el término chino para "extranjero", al contrario que "doméstico" (nei) o "interno"-.

\section{Conclusión}

En conclusión, podemos aprender de la biografía de Zhang Songxi que, a pesar de que existan sin duda cualidades internas y externas en las artes marciales, puede ser engañoso elevar las mismas a designaciones absolutas de escuelas 0 estilos.

\section{REFEREN CIAS}

Gu Yanwu 顾炎武 (1928)。《日知錄集釋》(Registro del conocimiento diario), 少 林僧兵 (Soldados monjes Shaolin). Shanghai: Zhonghua Shuju. Rollo 29, 21-22.

Henning, S. (1997). Chinese boxing: The internal versus external schools in the light of history and theory. Journal of Asian Martial Arts, 6(3): 11-19.

2 En su interpretación del código de las cinco palabras de Zhang Songxi, Shen Yiguan utiliza dos frases de la historia de la Doncella de Yue para explicar la intensidad y franqueza: "Comienza como una virgen", y "Después conviértete en una liebre asustada". 
Legge, J. (1961). The Chinese Classics: The works of Mencius, Book II, Part 1, Chapter II, 186. Taipei: W enxing Publishers. Publicado originalmente en 1892.

Takigawa, K. (1972). 瀧川龜太「《史記會注考證》(Registro de investigación histórica y apuntes). Taipei: Hongye Shuju.

Tang Hao 唐豪 (1969). 《内家拳的「究》 Neijia quande yanjiu (Investigación en el boxeo de la escuela interna). Hong Kong: Unicorn Press. Publicado originalmente en 1935.

Xie Zhaozhe 「肇浙 (1935).《五「「》(Cinco misceláneas). Beijing: Central Publishers. Rollo 5, 207.

Zhang Ru'an 「如安 (1988). 《「家拳大師張松溪生平辨誤》Neijiaquan dashi Zhang Songxi shengping bianwu (A clarando los malentendidos respecto a la vida del maestro Zhang Songxi de la escuela de boxeo interna). «体 育文史》 Physical Culture Literature and History, (4): 28-30.

Zhang Shouyong 張壽鏞, ed. (1932). 《四明叢書》 (Colección de trabajos de Si Ming [N ingbo]). Series 8, chapter 16.

Zi, Etienne (1971). Pratique des examens militaires en Chine. Varietes Sino-logiques, (9). Taipei: Cheng Wen. Publicado originalmente en 1896. Descargado de: http://libweb.uoregon.edu/ec/e-asia/read/.pdf.

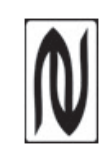

